\title{
A stretchable and adhesive ionic conductor based on polyacrylic acid and deep eutectic solvents
}

\author{
Gang Li ${ }^{1,5}$, Zhihao Deng ${ }^{1,5}$, Minkun Cai ${ }^{1}$, Kaixi Huang ${ }^{1}$, Mengxue Guo ${ }^{1}$, Ping Zhang ${ }^{2}$, Xingyu Hou ${ }^{1}$, Yuan Zhang ${ }^{1}$, Yueji Wang ${ }^{1}$, \\ Yan Wang ${ }^{1}$, Xiang $\mathrm{Wu}^{3}$ and Chuan Fei Guo $\mathbb{i D}^{1,4 凶}$
}

Hydrogels are a widely used ionic conductor in on-skin electronic and iontronic devices. However, hydrogels dehydrate in the open air and freeze at low temperatures, limiting their real applications when they are attached on skin or exposed to low temperatures. Polymer-ionic liquid gels can overcome these two obstacles, but synthetic ionic liquids are expensive and toxic. In this work, we present an ionic conductor based on polyacrylic acid (PAAC) and deep eutectic solvents (DESs) that well addresses the aforementioned challenges. We polymerize acrylic acid in DESs to get the PAAc-DES gel, which exhibits excellent stretchability $(>1000 \%)$, high electrical conductivity $\left(1.26 \mathrm{mS} \mathrm{cm}^{-1}\right)$, high adhesion to the skin $\left(\sim 100 \mathrm{~N} \mathrm{~m}^{-1}\right)$, as well as good anti-drying and anti-freezing properties. We also demonstrate that the PAAc-DES gel can be used as an on-skin electrode to record the surface electromyographic signal with high signal quality, or as a transparent stretchable electrode in iontronic devices that can work at $-20^{\circ} \mathrm{C}$. We believe that the PAAc-DES gels are an ideal candidate as epidermal electrodes or transparent stretchable electrodes.

npj Flexible Electronics (2021)5:23; https://doi.org/10.1038/s41528-021-00118-8

\section{INTRODUCTION}

Soft ionic conductors are a vital component in on-skin electronics that can be used as an interface to transfer electrical signals between the human body and the external devices ${ }^{1-5}$. A desired ionic conductor should exhibit high electrical conductance, high stretchability, as well as low cost for commercialization. Hydrogels are a promising material to conduct ions ${ }^{6-9}$, exhibiting skin-like mechanical performance, good biocompatibility, high electrical conductivity, and other attractive properties $^{3,8}$. Therefore, hydrogels have been applied in electrophysiological signal recording ${ }^{1}$, human-machine interaction ${ }^{10,11}$, ionic skins $^{12}$, and luminescent devices ${ }^{13,14}$. However, the properties of hydrogels are highly related to water content, while hydrogels dehydrate rapidly in open air. A dehydrated hydrogel loses its softness, stretchability and changes its shape. Therefore, additional seals or chemicals that prevent dehydration are often needed to prevent water loss 9 . In addition, hydrogels tend to freeze at a low temperature, which may hinder their applications in cold weather.

Polymer-ionic liquid gels (IL gels) are another selection of soft ionic conductors. lonic liquids (ILs) are salts of low melting point, and they are non-volatile at room temperature ${ }^{15,16}$, eliminating the problem of easy freezing and dehydration in hydrogels. As a result, the IL gels exhibit higher stability than the hydrogel. IL gels have demonstrated usefulness in various iontronic devices ${ }^{17-19}$. However, synthetic ILs are expensive and toxic, being major problems for the commercialization and on-skin applications of the IL gels ${ }^{20}$.

Deep eutectic solvents (DESs) are an emerging alternative to ILs, which are formed by mixing a quaternary ammonium salt (e.g., choline chloride, $\mathrm{ChCl}$ ) with another hydrogen bond donor (e.g., ethylene glycol, EG) ${ }^{21}$. DESs possess similar physico-chemical properties to those of common ILs, such as high electrical conductivity and low vapor pressure. The most profound advantage of DESs over ILs is their low cost and excellent biocompatibility ${ }^{20,22-24}$, and thus DES-based ionic conductors are rapidly developed. However, DES gels have not yet been comparable with common IL gels in terms of stretchability, transparency, and adhesive property ${ }^{25-27}$.

In this work, we show that a stretchable, transparent, adhesive, and anti-freezing DES gel can be prepared by polymerizing monomers in DESs, by mixing acrylic acid (AAC) with various DESs and initiating the reaction via ultraviolet (UV) radiation, similar to the method to make synthetic hydrogels. The prepared PAAc-DES gel is anti-drying and anti-freezing, demonstrating higher stability than the hydrogels. The gel also exhibits a high transparency of $\sim 92 \%$, a large stretchability over $1000 \%$, at moderate electrical conductivity of $1.26 \mathrm{mS} \mathrm{cm}^{-1}$, and strong adhesion $\left(\sim 100 \mathrm{~N} \mathrm{~m}^{-1}\right)$ with the human skin, which are comparable to most IL gels. The cost of the PAAC-DES gels, however, is about two orders of magnitudes lower than that of many synthetic ILs. The PAAc-DES gels also exhibit high compatibility on human skin without showing skin irritation after wearing for $8 \mathrm{~h}$. Thus, this soft ionic conductor has been applied as an on-skin electrode to record bioelectricity signals, exhibiting low impedance of the skin-electrode interface and improved signal quality compared with commercial electrodes. The anti-freezing PAAC-DES gel also can serve as an ionic skin working at $-20^{\circ} \mathrm{C}$. We believe that our work can enrich the ionic conductors with a cheaper, eco-friendly, and high-performance choice.

\section{RESULTS}

\section{Preparation and basic properties of PAAc-DES gel}

We mixed $\mathrm{ChCl}$ with four hydrogen bond donors including glycerol (Gly), urea, diethylene glycol (DG), and ethylene glycol (EG), all in a molar ratio of 1:2, to synthesize the four different DESs. The PAAC-DES gels were made by photo-curing monomer AAC,

\footnotetext{
${ }^{1}$ Department of Materials Science and Engineering, Southern University of Science and Technology, Shenzhen, China. ${ }^{2}$ Department of Mechanics and Aerospace Engineering, Southern University of Science and Technology, Shenzhen, China. ${ }^{3}$ School of Materials Science and Engineering, Shenyang University of Technology, Shenyang, Liaoning, China. ${ }^{4}$ Guangdong Provincial Key Laboratory of Functional Oxide Materials and Devices, Southern University of Science and Technology, Shenzhen, Guangdong, China. ${ }^{5}$ These authors contributed equally: Gang Li, Zhihao Deng. ${ }^{\circledR}$ email: guocf@sustech.edu.cn
} 

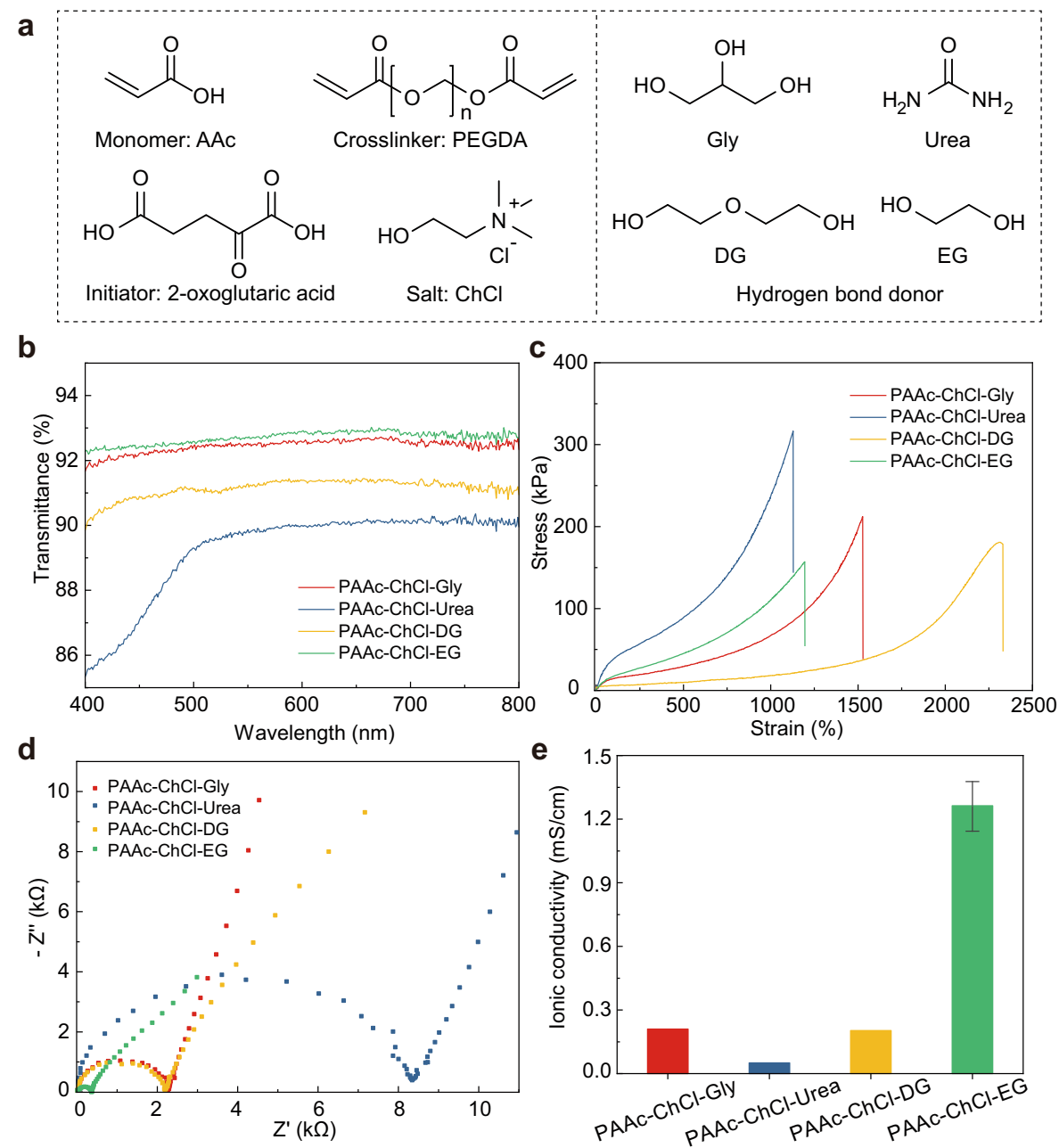

Fig. 1 Synthesis and optical, mechanical, and electrical properties of the PAAc-DES gels with different hydrogen bond donors. a Chemical structure of the monomer, crosslinker, initiator, salt, and four hydrogen bond donors. b UV-Vis spectrum of the four PAAc-DES gels. c Tensile stress-strain curves of the four PAAc-DES gels. d Nyquist plots and e ionic conductivity of the four PAAc-DES gels. Values in panel e represent the mean and standard deviation $(n=3)$. The standard deviations for PAAc-ChCl-Gly, PAAc-ChCl-Urea, and PAAc-ChCl-DG gels are not shown in the graph because they are negligible.

crosslinker poly(ethylene glycol) diacrylate (PEDGA), and initiator 2-oxoglutaric acid in DESs. The chemical structures of the molecules are shown in Fig. 1a. The precursor solution composes 28.9 wt.\% AAc and $70 \mathrm{wt} . \% \mathrm{DES}$, and the weight percentage of the crosslinker and initiator is $0.1 \%$ and $1 \%$, respectively. We clarify the four PAAC-DES gels according to their composition, such as PAAC-ChCl-Gly gel in this text.

The AAC is dissolved in all DESs to form clear solutions and all solutions are polymerized into a transparent gel (Fig. S1), indicating the high compatibility between the PAAC and the DESs. The UV-Vis spectra in Fig. $1 \mathrm{~b}$ show that the PAAc-ChCl-EG and the PAAc-ChCl-Gly gels have an optical transmittance above 92\%; the PAAc-ChCl-DG gel also exhibits a transmittance $>90 \%$, while only the PAAc-ChCl-Urea shows transparency $<90 \%$. The tensile properties of the four PAAC-DES gels are shown in Fig. 1c, showing that all the gels exhibit stretchability larger than $1000 \%$. Because of the different structures of the hydrogen bond donors, the modulus and strength of these gels are different. From Fig. S2, the PAAc-ChCl-Urea gel has the largest Young's modulus (50 \pm $4 \mathrm{kPa})$ and the highest tensile strength $(296 \pm 21 \mathrm{kPa})$. By comparison, the modulus of the PAAc-ChCl-DG gel is only $4 \mathrm{kPa}$, which is the softest one. The other two PAAc-DES gels have similar moduli of $\sim 20 \mathrm{kPa}$. All gels show moderate tensile strengths $>100 \mathrm{kPa}$. We also measured the ionic conductivity of these gels at room temperature. The Nyquist plots and the calculated ionic conductivities are given in Fig. 1d, e, respectively. The PAAC-ChCl-EG gel has the highest conductivity $(1.26 \pm$ $0.13 \mathrm{mS} \mathrm{cm}^{-1}$ ), whereas the PAAc-ChCl-Urea gel has the lowest conductivity $\left(0.05 \mathrm{mS} \mathrm{cm}^{-1}\right)$. Therefore, we chose the PAAC-ChCl-EG gel as the representative of the PAAc-DES gels to conduct further study for bio-electrical signal measurement and application in iontronic devices.

\section{Cyclic stretch-release and dynamic test}

The PAAc-ChCl-EG gel exhibits good recovery ability when it is released from elongation. Samples are subjected to loading-unloading tests at different strains, and the stress-strain curves are shown in Fig. 2a. The initial residual strain of the gel after releasing from $100 \%$ strain is about $20 \%$ and increases to $40 \%$ when the gel is stretched to $400 \%$. These samples are allowed to recover for $1 \mathrm{~min}$ and then subjected to a second stretch-release cycle. The stress-strain curves are shown in Fig. S3a-d, which indicate that the majority of the initial residual strain is removed. The sample is then tested over 100 cycles of loading-unloading, and the selected stress-strain curves are shown in Fig. S4. After 100 cycles of stretch-release, the stress at $100 \%$ strain reduces to $\sim 60 \%$ of its initial value, indicating that internal structures are partially broken. After $10 \mathrm{~min}$, the strength 


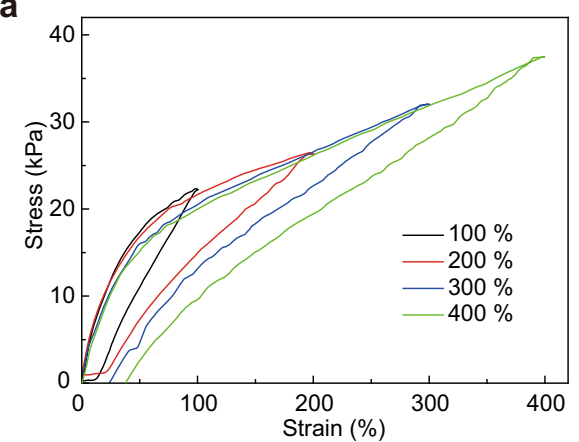

C

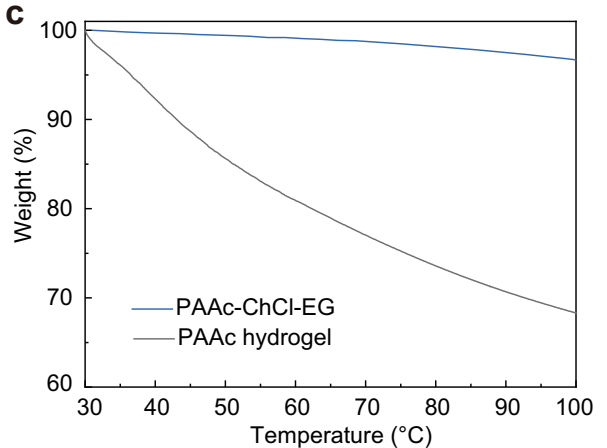

b

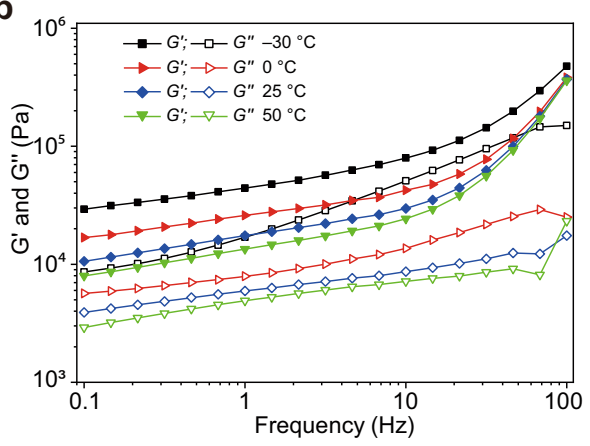

d

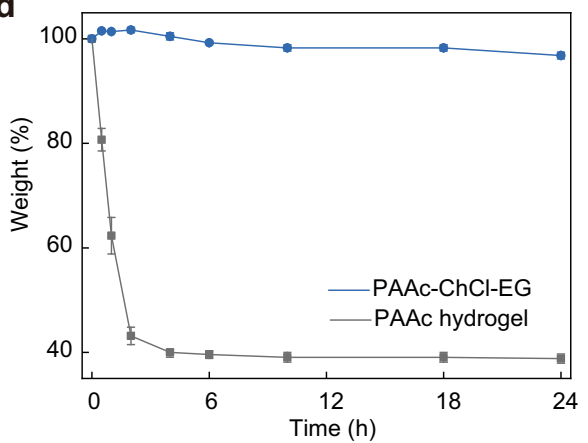

Fig. 2 Characterization of the PAAc-ChCl-EG gel. a Stress-strain curves of the gel in tension-unloading test with different elongations. b Storage modulus $\left(G^{\prime}\right)$ and loss modulus $\left(G^{\prime \prime}\right)$ of the gel in different frequencies and at different temperatures. $c$ TGA curve of the PAAc-ChCl-EG and the PAAc hydrogel. d Evolution of mass with the time of the PAAc-ChCl-EG and PAAc hydrogel at $37^{\circ} \mathrm{C}$. Values in panel $d$ represent the mean and standard deviation $(n=3)$.

of the gel restores to $\sim 75 \%$ of its initial value and the residual strain reduces to $4 \%$. Because the ionic conductor serves as a stretchable electrode, the important consideration is the dimension stability instead of strength. In this sense, the PAAc-ChCl-EG gel performs well because it has a small residual strain after being elongated to large strains or being stretched for 100 cycles, enabling possible applications as a stretchable electrode.

The dynamic mechanical properties of the PAAc-ChCl-EG gel at different temperatures are shown in Fig. 2b, which exhibit frequencydependent storage modulus $\left(G^{\prime}\right)$ and loss modulus $\left(G^{\prime \prime}\right)$ in the temperature range of $-30-50^{\circ} \mathrm{C}$. The storage modulus becomes larger as the oscillating frequency increases. For instance, at room temperature, $G^{\prime}$ of the gel is $10.6 \mathrm{kPa}$ at $0.1 \mathrm{~Hz}$, and it reaches $368.7 \mathrm{kPa}$ at $100 \mathrm{~Hz}$. This is because that the gel contains high solid content, and the entanglement of polymer chains contributes more to the $G^{\prime}$ at a shorter time scale, or higher frequency. Another observation is that the $G^{\prime}$ at $0.1 \mathrm{~Hz}$ of the gel only increases to $18.6 \mathrm{kPa}$ at $-30^{\circ} \mathrm{C}$ compared with the one measured at $25^{\circ} \mathrm{C}$. That means, the gel becomes two times harder after it drops from room temperature to an ultralow temperature of $-30^{\circ} \mathrm{C}$, at which most hydrogels freeze to have a rigidity increase of $\sim 5$ orders of magnitude.

\section{Thermostability}

We tested the thermal stability of the gel using thermogravimetric analysis (TGA), and the result is shown in Fig. 2c. The PAAC-ChCl-EG gel loses only $\sim 4 \%$ of its initial mass at $100{ }^{\circ} \mathrm{C}$, indicating that the gel can be used at a relatively high temperature. For comparison, we prepared a PAAC hydrogel containing the same content of PAAc and conducted the same test, as shown in Fig. 2c. The hydrogel starts to lose water as the test begins, and the mass of the hydrogel reduces to $70 \%$ when the temperature reaches $100^{\circ} \mathrm{C}$. We measured the weight change of the PAAC-ChCl-EG gel and the PAAc hydrogel at $37^{\circ} \mathrm{C}$ for $24 \mathrm{~h}$ and it is plotted in Fig. $2 \mathrm{~d}$. The hydrogel loses $57 \%$ of the water within $2 \mathrm{~h}$ and has a weight loss of $\sim 60 \%$ over $24 \mathrm{~h}$. The evaporation of water from the hydrogel results in significant shrinkage in dimensions, as shown in Fig. S5. In contrast, the weight of the PAAc-ChCl-EG gel maintains stability throughout the test. In the first few hours, the weight change of the PAAc-ChCl-EG gel is about $1 \%$, and after $24 \mathrm{~h}$, the PAAc-ChCl-EG gel loses $\sim 3 \%$ of its mass due to the slow evaporation of EG. These results indicate a far higher thermostability of the PAAC-DES gel than hydrogels.

\section{Comparison of the PAAC-DES gel with other IL gels and DES gels}

The performances of the PAAC-DES gel are compared with those of other representative non-water ionic conductors in Table 1. Our PAAC-DES gel is comparable to other materials in terms of transparency, but one order of magnitude higher than that of other DES gels in stretchability $(\lambda)$. In addition, the stretchability and electrical conductivity of our PAAc-DES gel are comparable to that of most IL gels. Price is another great concern in practical applications, and our PAAc-DES gel is superior in this consideration due to the low price of the chemicals used. The price is $<\$ 10 \mathrm{~kg}^{-1}$ for $\mathrm{ChCl}$ and $\sim \$ 1 \mathrm{~kg}^{-1}$ for EG, which are several orders of magnitude lower than that of most synthetic ionic liquids (Table S1). In this sense, our DES gels are a better choice than IL gels since they present a similar performance with ILs but have a much lower cost.

\section{Adhesion of the PAAc-DES gel and its application as an on- skin electrode}

The PAAc-ChCl-EG can adhere to various substrates, such as metals and the human skin. Figure. 3a shows that a piece of PAAc-ChCl-EG that is adhered to two copper strips. When we pulled the two copper strips away to a large tensile strain up to $800 \%$, the PAAC-DES gel-copper interface is tough enough to prevent the separation of the two materials. We measured the 
Table 1. Comparison of our work with representative IL gels and DES gels.

\begin{tabular}{|c|c|c|c|c|c|c|}
\hline Type & Composition (polymer and solvent) & $T(\%)$ & $\lambda(\%)$ & $\sigma\left(\mathrm{mS} \mathrm{cm}^{-1}\right)$ & Price & Ref. \\
\hline & PEA and [BMMIm][TFSI] & 93.6 & $>1000$ & $0.01-1$ & Expensive & 18 \\
\hline & $\mathrm{P}(\mathrm{VDF}-\mathrm{HFP}) \& \mathrm{P}(\mathrm{MMA}-\mathrm{BMA})$ and [EMIM][TFSI] & 93 & 307 & $>1$ & Expensive & 28 \\
\hline \multirow[t]{2}{*}{ DES gel } & Gelatin and [ChCl][EG] & $80-90$ & $>300$ & 2.5 & Very cheap & 25 \\
\hline & PAAm and [ChCl][urea, glycerol] & - & $>200$ & $\sim 1$ & Very cheap & 26 \\
\hline
\end{tabular}

interfacial toughness by $180^{\circ}$ peeling, as shown in Fig. $3 \mathrm{~b}$ and Fig. S6. The toughness values between PAAc-ChCl-EG gel and copper, steel, and tin are $109 \pm 12 \mathrm{~N} \mathrm{~m}^{-1}, 129 \pm 10 \mathrm{~N} \mathrm{~m}^{-1}$, and $85 \pm 9 \mathrm{~N} \mathrm{~m}^{-1}$, respectively. The gel is also highly adhesive to the human skin. Fig. $3 c$ shows that when the free end of a gel sample undergoes large deformation, the other end is still well adhered to the skin and causes large skin deformation. In Fig. 3d, we compare the adhesive performance of the PAAc-ChCl-EG gel with that of commercial $3 \mathrm{M}$ VHB tapes. The interfacial toughness between our gel and the skin is around $100 \mathrm{~N} \mathrm{~m}^{-1}$, which is larger than that of the skin-VHB tape interface $\left(<70 \mathrm{~N} \mathrm{~m}^{-1}\right)$. The strong adhesion of the PAAc-DES gel is originated from the abundant $-\mathrm{COOH}$ function groups of PAAC, which interact with the adherend by Van der Waals force. Another contribution to the strong adhesion is the viscoelasticity of the PAAc-ChCl-EG achieved by tuning the crosslinking density ${ }^{28}$. This can be evidenced in Fig. S7, showing that both the $G^{\prime}$ and $G^{\prime \prime}$ of the PAAc-ChCl-EG gel are strongly dependent on the density of crosslinking. If no crosslinker is added, the linear PAAC and DES form a sticky product and a residue will be left on the skin after removing the gel. By contrast, if crosslinking density is too high, the gel is no longer adhesive (Fig. S8). We further examined the compatibility of the DES gel with skin. One volunteer subject wore a piece of PAAc-ChCl-EG gel on his forearm for over $8 \mathrm{~h}$, and the gel remained adhesive. After the sample was removed, the skin showed no sign of irritation, as shown in Fig. 3e, $\mathrm{f}$.

We used our PAAc-ChCl-EG gel as surface electrodes and compared our gel with commercial $\mathrm{Ag}-\mathrm{AgCl}$ electrodes serving as control samples. The PAAc-ChCl-EG gel was first cut in the same dimensions as the commercial $\mathrm{Ag}-\mathrm{AgCl}$ electrodes and then adhered to both our electrodes and the control samples were attached to the biceps brachii. The impedance values $(|Z|)$ of both electrodes were measured and the results are provided in Fig. $3 \mathrm{~g}$. Over most of the frequency range, our gel has a lower impedance than that of the commercial one. For instance, the $|Z|$ of the DES gel is $173 \mathrm{k} \Omega$ at $10 \mathrm{~Hz}$ and $4.4 \mathrm{k} \Omega$ at $1 \mathrm{kHz}$, while those for the commercial electrode are $238 \mathrm{k} \Omega$ and $12.3 \mathrm{k} \Omega$, respectively. The low impedance lies in the high electrical conductance of our gel and the conformal contact between the PAAc-ChCl-EG gel and $\mathrm{skin}^{4}$. Electromyographic (EMG) signals recorded using the DES gel electrodes and commercial electrodes are shown in Fig. 3h. The signals are generated from the same action of squeezing a hand gripper by the same person. The amplitudes of both EMG signals are almost the same, but the one recorded from our electrodes has a lower noise level than that recorded from the $\mathrm{Ag}-\mathrm{AgCl}$ electrodes (Fig. S9). The improved EMG signal quality is believed to be related to the lower impedance of the PAAc-DES gels, which might be extended to the collection of other bioelectricity signals.

\section{Anti-freezing ionic skin}

The PAAC-ChCl-EG gel possesses a high anti-freezing performance, which allows the gel to work under low temperatures down to $-60^{\circ} \mathrm{C}$. Fig. $4 a$ presents the differential scanning calorimetric (DSC) curve of the PAAc-ChCl-EG gel that is cooling from $20^{\circ} \mathrm{C}$ to $-60^{\circ} \mathrm{C}$, showing a flat curve that indicates no glass transition in this temperature range. For comparison, an exothermic peak located near $-15^{\circ} \mathrm{C}$ has appeared in the DSC curve of the PAAc hydrogel, indicating that the hydrogel begins to freeze at this temperature. In Fig. S10, we show photographs of the PAAC hydrogel and the PAAc-DES gel kept at $-60^{\circ} \mathrm{C}$. The hydrogel is frozen into a white and stiff solid, whereas the PAAc-ChCl-EG gel remains transparent. We pulled both specimens at $-60^{\circ} \mathrm{C}$, and the frozen hydrogel lost its stretchability, while the PAAc-ChCl-EG gel remained soft and stretchable. We thus anticipate that the PAAc-ChCl-EG gel can be used in extremely low-temperature environments, including the Antarctic Pole. Besides, it is worth noting that the glass transition temperature of the PAAc-ChCl-EG gel is lower than that of the pure PAAC $\left(\sim 130^{\circ} \mathrm{C}\right.$, see Fig. S11) and the freezing temperature of $\mathrm{ChCl}-\mathrm{EG}\left(-36^{\circ} \mathrm{C}\right)^{29}$. This anti-freezing behavior may be attributed to the interaction between the PAAC and the ChCl-EG, in which the PAAc hinders the crystallization of the solvent and the solvent also softens the polymer chains. After all, this observation needs further work to provide a valid explanation.

The high stretchability of the PAAc-DES gel under low temperatures allows for applications in anti-freezing ionic skin. The device structure of the ionic skin is illustrated in Fig. 4b, with two DES gel electrodes sandwiching an insulating VHB tape serving as the dielectric ${ }^{12}$. A photograph of this ionic skin is shown in Fig. S12, in which the gel is dyed for better distinction. The sensitivity of the ionic skin under pressing is $5 \mathrm{MPa}^{-1}$, and the gauge factor under stretch is 0.42 (Fig. 4c, d); both are comparable to the previous results ${ }^{25}$. Upon pressing, the sensor can respond to the stimuli both at room temperature and at $-20^{\circ} \mathrm{C}$, as shown in Fig. $4 \mathrm{e}, \mathrm{f}$, suggesting that the ionic skin can work at $-20^{\circ} \mathrm{C}$. However, the signal intensity at $-20^{\circ} \mathrm{C}$ is weaker than that at room temperature because of the increased rigidity of the gel at a lower temperature. For other hydrogel-based ionic skins, the whole device will lose sensing function at such a low temperature. We also demonstrated that the PAAc-ChCl-EG gel can be used in antifreezing iontronic luminescence that works at a temperature lower than $-16^{\circ} \mathrm{C}$ (Fig. S13). Low-temperature tolerance is vital for onskin electronics, especially in outdoor applications. Here our results indicate that the PAAC-DES gel is a promising electrode for anti-freezing iontronics under extremely low-temperature environments.

\section{DISCUSSION}

In this work, we demonstrated the great potential of the PAAC-DES gel in the field of on-skin electrodes and iontronics devices. The PAAC-DES gel is fabricated by UV curing AAC in DES. The obtained PAAc-DES gels show high stretchability, transparency, and adhesive performance that are comparable with hydrogels and IL gels. Our PAAC-DES gels present the advantage of non-volatility and anti-freezing ability, as well as low price. Such advantages make the PAAc-DES gels a superior alternative to IL 
a

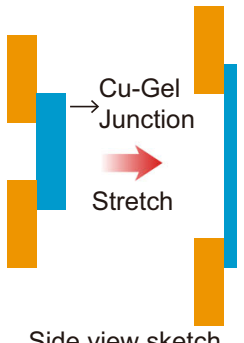

Side view sketch

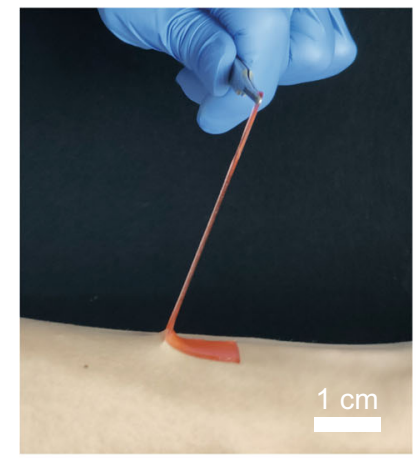

e

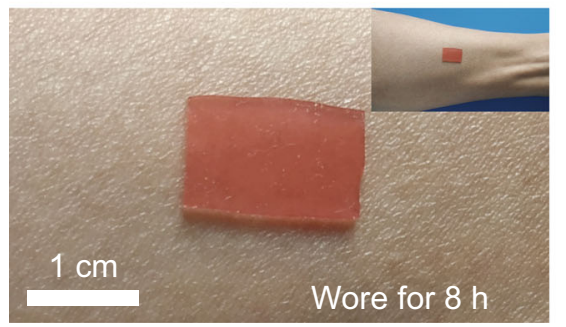

g

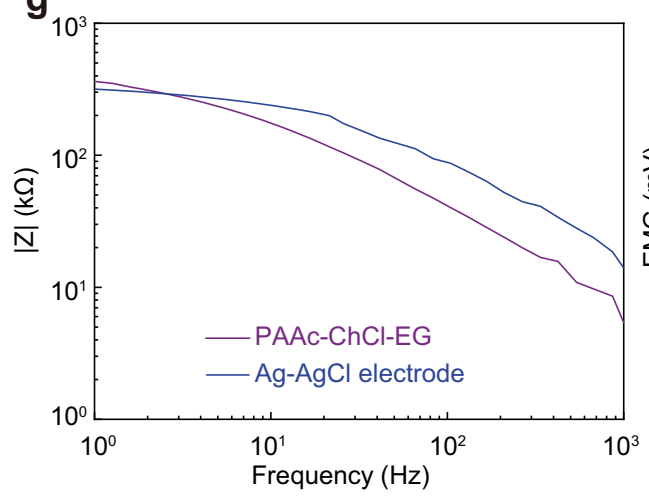

b
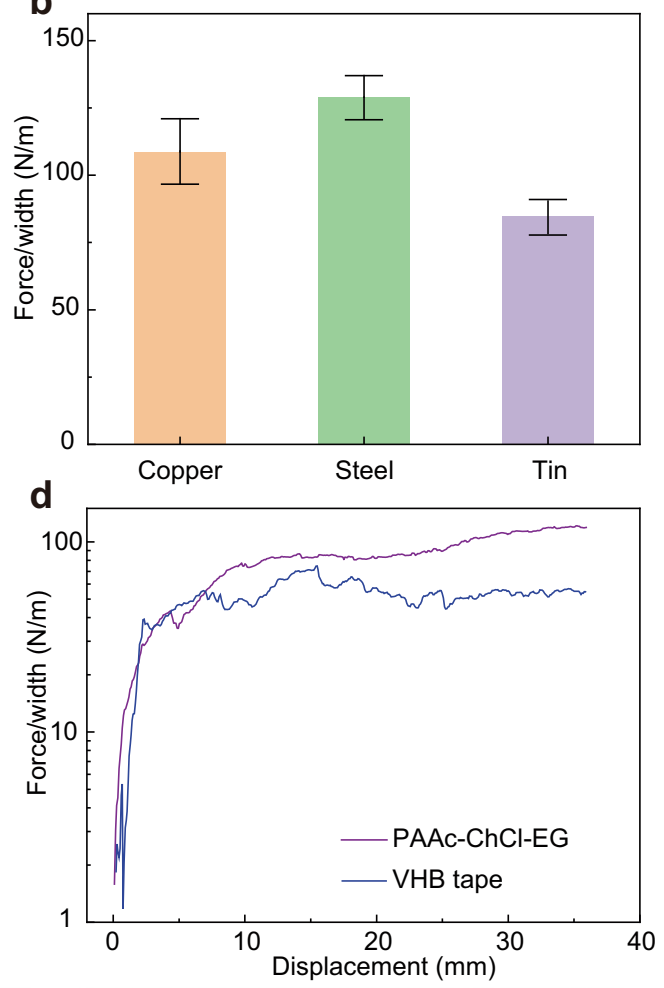

f

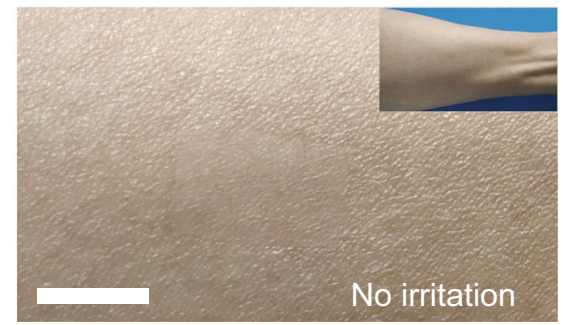

h

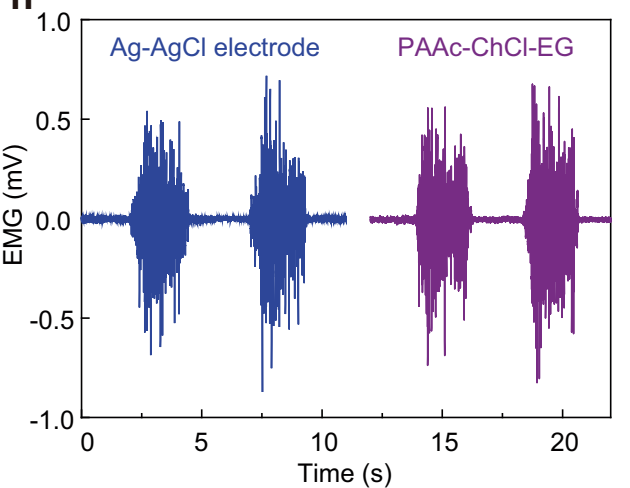

Fig. 3 Adhesive performance of the PAAc-ChCl-EG gel and its application for on-skin electrodes. a Sketch and photograph using the PAAc-ChCl-EG gel to adhere two copper strips. $\mathbf{b}$ The measured peeling forces per width of the PAAc-ChCl-EG gel on copper, steel, and tin. c Photograph of pulling a PAAc-ChCl-EG gel away from the skin of a forearm. $\mathbf{d}$ Comparison of the adhesion behavior of the PAAc-ChCl-EG gel with a VHB tape. e Photograph of the gel attached on the skin for $8 \mathrm{~h}$. $\mathbf{f}$ Photograph of the skin after removing the PAAC-ChCl-EG gel. $\mathbf{g}$ Impedances of the PAAc-ChCl-EG gel and the $\mathrm{Ag}-\mathrm{AgCl}$ electrode on the skin. $\mathbf{h}$ Recorded EMG signals using DES gel electrode and $\mathrm{Ag}-\mathrm{AgCl}$ electrode. Values in panel $\mathbf{b}$ represent the mean and standard deviation $(n=3)$.

gels. We have shown that our gel can be used as an on-skin electrode to record surface EMG signals and to construct ionic skins that can sense external stimuli at low temperatures down to $-20^{\circ} \mathrm{C}$. Such low-temperature applications of the PAAc-DES gels may be extended to other devices not demonstrated in this work. We believe that the PAAc-DES gels are a promising alternative to the hydrogels and IL gels in various circumstances.

\section{METHODS}

\section{Materials}

Choline chloride (ChCl), glycerol (Gly), diethylene glycol (DG), ethylene glycol (EG), urea, acrylic acid (AAc), poly(ethylene glycol) diacrylate (PEGDA, Mw. 600), and 2-oxoglutaric acid were purchased from Shanghai Aladdin Biochemical Technology Co., Ltd. Silicon rubber (Sylgard 184) was 
a
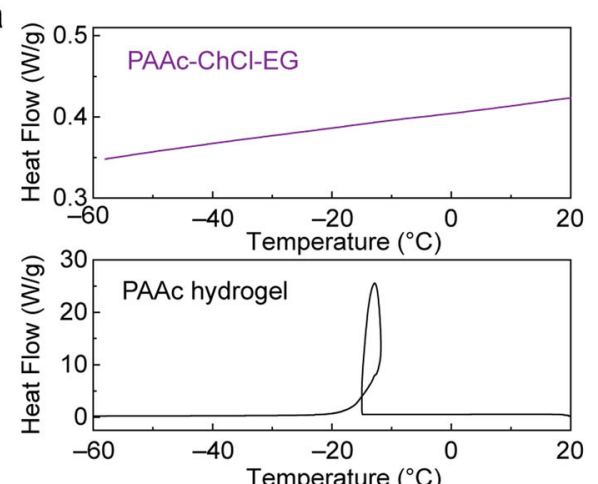

C

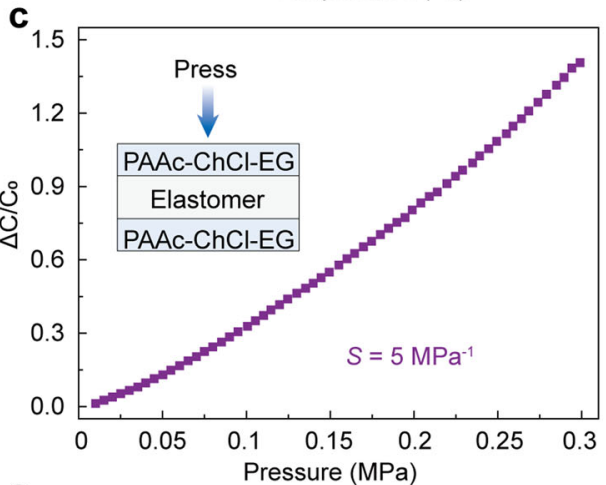

e

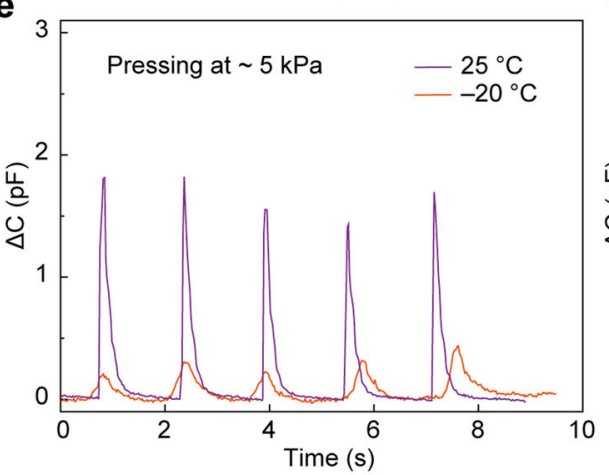

b

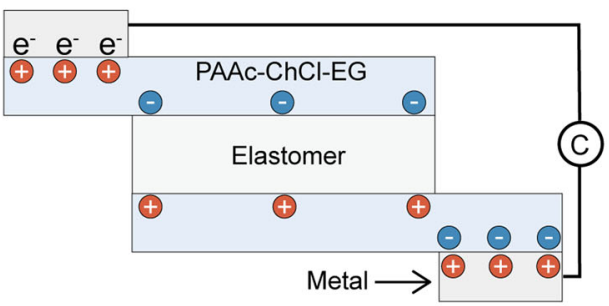

Structure of ionic skin

d

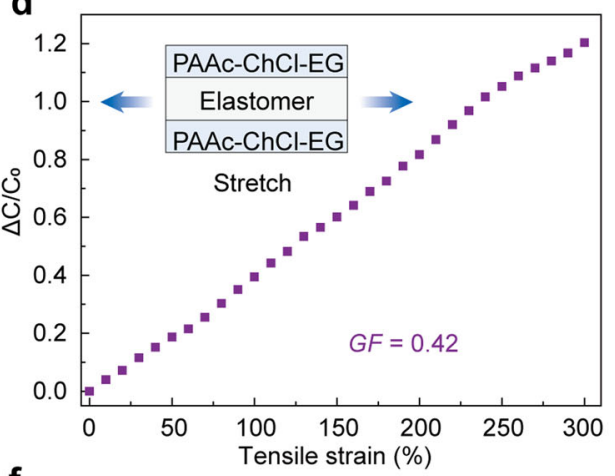

f

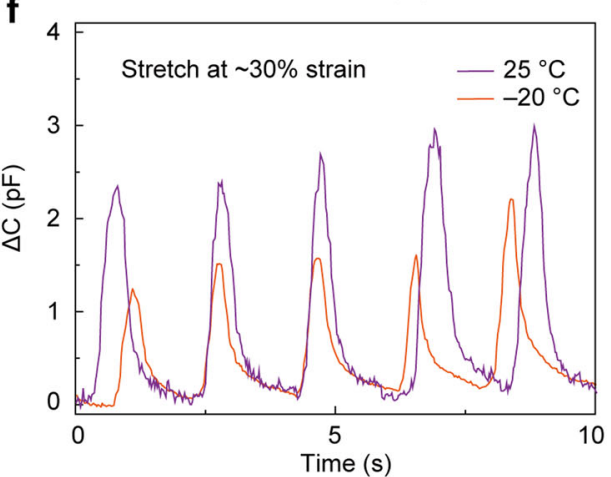

Fig. 4 PAAC-DES gel-based ionic skin for low-temperature applications. a Differential scanning calorimetry (DSC) curves of the PAAC-ChCl-EG gel and the PAAc hydrogel that are cooling from 20 to $-60^{\circ} \mathrm{C}$. b Illustration of the device structure. c Response of the ionic skin as a function of applied pressure. $\mathbf{d}$ Response of the ionic skin to different tensile strains. e Signal generated by finger touching at room temperature and $-20^{\circ} \mathrm{C}$, respectively. $f$ Signal generated by stretching at room temperature and $-20^{\circ} \mathrm{C}$, respectively.

purchased from DOWSIL. ZnS:Cu particles were purchased from Shanghai Keyan Co. China. All reagents were used as received.

\section{Preparation of PAAc-DES gel}

The DESs were prepared by mixing $\mathrm{ChCl}$ with hydrogen bond donors, including Gly, urea, $\mathrm{DG}$, and $\mathrm{EG}$, in a molar ratio of 1:2. The mixtures were then stirred at $100^{\circ} \mathrm{C}$ for $2 \mathrm{~h}$. Next, $2.89 \mathrm{~g} \mathrm{AAc}, 7 \mathrm{~g} \mathrm{DES}, 0.1 \mathrm{~g}$ 2-oxoglutaric acid, and $0.01 \mathrm{~g}$ PEGDA were added into a vial, and VORTEX 3 was used to accelerate dissolution. The PAAc-DES precursor was injected into a glass mold separated by spacers, and then the glass mold was placed under a $365 \mathrm{~nm}$ UV radiation for $3 \mathrm{~min}$. A PAAc hydrogel was prepared using the same steps by changing DES to water.

\section{Basic properties characterization}

The UV-Vis spectra of the PAAc-DES gels were recorded by the PE LAMBDA 950 instrument in the range of $400-800 \mathrm{~nm}$. The thickness of the sample was $1 \mathrm{~mm}$, and the air was taken as a reference.

The tensile properties of the PAAc-DES gels were measured by a universal testing machine (XLD-20E, Jingkong Mechanical testing Co., Ltd). The specimens were cut into a dumbbell shape $(35 \mathrm{~mm}$ in total length,
$2 \mathrm{~mm}$ in inner width, and $12 \mathrm{~mm}$ in gauge length) and stretched at a speed of $100 \mathrm{~mm} \mathrm{~min}^{-1}$.

The bulk resistance $R_{\mathrm{b}}$ of the PAAc-DES gel was measured by the AC impedance spectroscopy measurements (CS series, Wuhan CorrTest Instruments Corp., Ltd.). The ionic conductivities $\sigma$ of the PAAc-DES gels were calculated by the formula $\sigma=L / S R_{\mathrm{b}}$, where $L$ and $S$ are the thickness and cross-section area of the specimen, respectively.

The rheology property of the PAAc-ChCl-EG gel was measured by a HAAKE MARS III rheometer using a $20 \mathrm{~mm}$ parallel plate geometry. Frequency sweep was measured at a shear strain of $1 \%$ at different temperatures.

Thermostability of the PAAc-ChCl-EG gel and PAAc hydrogel was measured by a thermogravimetric analyzer (Discovery TGA) at a heating rate of $10 \mathrm{~K} \mathrm{~min}^{-1}$ under nitrogen atmosphere.

The weight change of the PAAc-ChCl-EG gel and PAAc hydrogel at $37^{\circ} \mathrm{C}$ was recorded at different time intervals; after the weighing, the samples were stored in an oven at a pre-set temperature.

The thermal behavior of the PAAc-ChCl-EG gel and PAAc hydrogel were studied by differential scanning calorimetry (DSC). About $5 \mathrm{mg}$ of the PAAc-ChCl-EG gel and PAAc hydrogel were put in the pan and heated or cooled at a speed of $10 \mathrm{~K} \mathrm{~min}^{-1}$ in nitrogen in a Discovery DSC. 
The $90^{\circ}$ and $180^{\circ}$ peeling tests were conducted by cutting the specimen are cut into a dimension of $100 \times 10 \times 1 \mathrm{~mm}^{3}$ and glued to a PI tape as stiff backing to prevent elongation during testing. The test speed was $50 \mathrm{~mm} \mathrm{~min}^{-1}$.

\section{Impedance measurement and EMG recording}

A pair of the electrodes (the PAAc-ChCl-EG gel or the Ag-AgCl gel electrodes (CH50RB, Nison Instrument (Shanghai) Limited)) adhered on biceps brachii separated by a distance of $2 \mathrm{~cm}$. The impedance was measured by an electrochemical workstation (CS series, Wuhan CorrTest Instruments Corp., Ltd.) from $1 \mathrm{~Hz}$ to $10 \mathrm{kHz}$.

Electromyographic (EMG) signals were recorded using the micro-4 system (Cambridge Electronic Design Limited) in a frequency range from 1 to $1000 \mathrm{~Hz}(50 \mathrm{~Hz}$ is filtered). Two working electrodes were attached to the same position for impedance measurement; a reference electrode was placed at the elbow. The action was squeezing a hand gripper.

\section{lonic skin fabrication and measurement}

Two pieces of PAAc-ChCl-EG gels were adhered to both sides of a $3 \mathrm{M} \mathrm{VHB}$ tape; one end of the PAAC-ChCl-EG gel was connected to a silver wire. The capacitance signal was obtained from a Keysight E4980AL LCR meter, the working frequency was $10 \mathrm{kHz}$.

\section{Fabrication of iontronic luminescence}

$\mathrm{ZnS}: \mathrm{Cu}$ particles were mixed with the PDMS base and curing agent in a weight ratio of 1:10:1. The mixture was spin coated on a piece of glass at $300 \mathrm{rpm}$ for $1 \mathrm{~min}$, and then heated at $65^{\circ} \mathrm{C}$ for $4 \mathrm{~h}$, forming the phosphor layer. The device was fabricated by sandwiching the phosphor layer between two pieces of PAAc-ChCl-EG gel. Copper foils were attached onto the DES gel to connect the power source (Trek 615). The cold environment is provided by a cold trap and the temperature is measured by using an Infrared camera ( $226 \mathrm{~s}$, Fotric, USA).

\section{Experiments on human subjects}

All experiments were conducted under approval from the Institutional Review Board at the Southern University of Science and Technology (protocol number: 20190007).

\section{Reporting summary}

Further information on research design is available in the Nature Research Reporting Summary linked to this article.

\section{DATA AVAILABILITY}

The experimental data referenced in this text are available from the authors upon reasonable request.

Received: 1 May 2021; Accepted: 29 July 2021; Published online: 23 August 2021

\section{REFERENCES}

1. Pan, L. et al. A compliant ionic adhesive electrode with ultralow bioelectronic impedance. Adv. Mater. 32, 2003723 (2020).

2. Yuk, H., Lu, B. \& Zhao, X. Hydrogel bioelectronics. Chem. Soc. Rev. 48, 1642-1667 (2019).

3. Liu, X., Liu, J., Lin, S. \& Zhao, X. Hydrogel machines. Mater. Today 36, 102-124 (2020).

4. Lopes, P. A. et al. Soft bioelectronic stickers: selection and evaluation of skininterfacing electrodes. Adv. Healthc. Mater. 8, 1900234 (2019).

5. $\mathrm{Wu}, \mathrm{H}$. et al. Materials, devices, and systems of on-skin electrodes for electrophysiological monitoring and human-machine interfaces. Adv. Sci. 8, 2001938 (2021).

6. Rong, Q., Lei, W. \& Liu, M. Conductive hydrogels as smart materials for flexible electronic devices. Chem. Eur. J. 24, 16930-16943 (2018).

7. Yang, C. \& Suo, Z. Hydrogel ionotronics. Nat. Rev. Mater. 3, 125-142 (2018).

8. Lee, Y., Song, W. J. \& Sun, J. Y. Hydrogel soft robotics. Mater. Today Phys. 15, 100258 (2020).
9. Zhang, D. et al. Self-powered ionic sensors overcoming the limitation of ionic conductors as wearable sensing devices. Mater. Today Phys. 15, 100246 (2020).

10. Zhang, Q., Liu, X., Duan, L. \& Gao, G. Nucleotide-driven skin-attachable hydrogels toward visual human-machine interfaces. J. Mater. Chem. A. 8, 4515-4523 (2020).

11. Chen, L. et al. 3D printed super-anti-freezing self-adhesive human-machine interface. Mater. Today Phys. 19, 100404 (2021).

12. Sun, J. Y., Keplinger, C., Whitesides, G. M. \& Suo, Z. Ionic skin. Adv. Mater. 26, 7608-7614 (2014).

13. Yang, $C$. et al. lonotronic luminescent fibers, fabrics, and other configurations. Adv. Mater. 32, 2005545 (2020).

14. Yang, C. H. et al. Electroluminescence of giant stretchability. Adv. Mater. 28, 4480-4484 (2016).

15. Galiński, M., Lewandowski, A. \& Stępniak, I. Ionic liquids as electrolytes. Electrochim. Acta. 51, 5567-5580 (2006).

16. Dong, K. et al. Multiscale studies on ionic liquids. Chem. Rev. 117, 6636-6695 (2017).

17. Lai, J. et al. Highly stretchable, fatigue-resistant, electrically conductive, and temperature-tolerant ionogels for high-performance flexible sensors. ACS Appl. Mater. Interfaces 11, 26412-26420 (2019).

18. Shi, L. et al. Highly stretchable and transparent ionic conductor with novel hydrophobicity and extreme-temperature tolerance. Research 2020, 2505619 (2020).

19. Lei, Z. \& Wu, P. A highly transparent and ultra-stretchable conductor with stable conductivity during large deformation. Nat. Commun. 10, 1-9 (2019).

20. Zhang, Q., Vigier, K. D. O., Royer, S. \& Jerome, F. Deep eutectic solvents: syntheses, properties and applications. Chem. Soc. Rev. 41, 7108-7146 (2012).

21. Paiva, A. et al. Natural deep eutectic solvents - solvents for the 21st century. ACS Sustain. Chem. Eng. 2, 1063-1071 (2014).

22. Smith, E. L., Abbott, A. P. \& Ryder, K. S. Deep eutectic solvents (DESs) and their applications. Chem. Rev. 114, 11060-11082 (2014).

23. Macário, I. et al. Cytotoxicity profiling of deep eutectic solvents to human skin cells. Sci. Rep. 9, 1-9 (2019).

24. Crump, M. R. et al. Sensorized tissue analogues enabled by a 3D-printed conductive organogel. npj Flex. Electron. 5, 1-8 (2021).

25. Qin, H., Owyeung, R. E., Sonkusale, S. R. \& Panzer, M. J. Highly stretchable and nonvolatile gelatin-supported deep eutectic solvent gel electrolyte-based ionic skins for strain and pressure sensing. J. Mater. Chem. C. 7, 601-608 (2019).

26. Hong, S. et al. A stretchable and compressible ion gel based on a deep eutectic solvent applied as a strain sensor and electrolyte for supercapacitors. J. Mater. Chem. C. 8, 550-560 (2020).

27. Qin, H. \& Panzer, M. J. Chemically cross-linked poly (2-hydroxyethyl methacrylate)-supported deep eutectic solvent gel electrolytes for eco-friendly supercapacitors. ChemElectroChem 4, 2556-2562 (2017).

28. Gu, Z. et al. Skin adhesives with controlled adhesion by polymer chain mobility. ACS Appl. Mater. Interfaces 11, 1496-1502 (2018).

29. Hansen, B. B. et al. Deep eutectic solvent: a review of fundamentals and applications. Chem. Rev. 121, 1232-1285 (2021).

\section{ACKNOWLEDGEMENTS}

The work was funded by the National Natural Science Foundation of China (No. $52073138,51903118)$, the "Guangdong Innovative and Entrepreneurial Research Team Program" under Contract No. 2016ZT06G587, the "Science Technology and Innovation Committee of Shenzhen Municipality" (Grant No. JCYJ20170817111714314), the Shenzhen Sci-Tech Fund (No. KYTDPT20181011104007) and Guangdong Provincial Key Laboratory Program (2021B1212040001) from the Department of Science and Technology of Guangdong Province. Y. Wang thanks the support of 2020 National College Students' Innovation Training Program under Contract No. 2020 S13.

\section{AUTHOR CONTRIBUTIONS}

G.L., Z.D., and C.F.G. designed the project and experiments. G.L. and Z.D. fabricated the materials and conducted the characterization. M.C. tested the performance of the ionic skin. P.Z. fabricated the iontronic luminescence. All authors discussed the results and commented on the manuscript.

\section{COMPETING INTERESTS}

The authors declare no competing interests.

\section{ADDITIONAL INFORMATION}

Supplementary information The online version contains supplementary material available at https://doi.org/10.1038/s41528-021-00118-8. 
Correspondence and requests for materials should be addressed to C.F.G.

Reprints and permission information is available at http://www.nature.com/ reprints

Publisher's note Springer Nature remains neutral with regard to jurisdictional claims in published maps and institutional affiliations. (c) Open Access This article is licensed under a Creative Commons Attribution 4.0 International License, which permits use, sharing, adaptation, distribution and reproduction in any medium or format, as long as you give appropriate credit to the original author(s) and the source, provide a link to the Creative Commons license, and indicate if changes were made. The images or other third party material in this article are included in the article's Creative Commons license, unless indicated otherwise in a credit line to the material. If material is not included in the article's Creative Commons license and your intended use is not permitted by statutory regulation or exceeds the permitted use, you will need to obtain permission directly from the copyright holder. To view a copy of this license, visit http://creativecommons. org/licenses/by/4.0/.

(c) The Author(s) 2021 